\title{
Can Localized Stenosis of the Main Pancreatic Duct be a Predictive Factor for Early Detection of Pancreatic Cancer?
}

\author{
Mamoru Takenaka, Kentaro Yamao and Masatoshi Kudo \\ Department of Gastroenterology and Hepatology, Kindai University Faculty of Medicine, Osaka, Japan
}

See "Predictive Value of Localized Stenosis of the Main Pancreatic Duct for Early Detection of Pancreatic Cancer" by Yoshihide Kanno, Shinsuke Koshita, Takahisa Ogawa, et al., on page 588-597.

We would like to comment on the study by Kanno et al. investigating the predictive value of a single localized stenosis of the main pancreatic duct (MPD) for the diagnosis of early-stage pancreatic cancer. ${ }^{1}$ Pancreatic cancer has a poor prognosis, and early-stage diagnosis is often challenging for anatomical reasons. However, the 5-year survival rate for patients with tumors measuring $\leq 10 \mathrm{~mm}$ is approximately $80 \%{ }^{2}$ Therefore, early diagnosis is vital for improving patient outcomes.

In the current study, Kanno et al. focused on a single, localized stenosis of the MPD without an identifiable mass as a useful finding for the early detection of pancreatic cancer. ${ }^{1}$ Localized stenosis was defined as an obvious decrease in MPD diameter compared with the diameters of both the ampullary and tail sides of the MPD. They called this findings for indicating suspicious pancreatic cancer at an early stage ("FiCE") and assessed its predictive value in the early detection of pancreatic cancer. Among 689 patients who underwent endoscopic retrograde pancreatography from January 2008 to September 2018 in the Sendai City Medical Center Japan, 19 patients with FiCE were enrolled. Patients with a detectable mass

Received: October 26, 2019 Accepted: October 29, 2019

Correspondence: Mamoru Takenaka

Department of Gastroenterology and Hepatology, Kindai University Faculty of Medicine, 377-2 Ohnohigashi, Osaka-Sayama City, Osaka 589-8511, Japan Tel: +81-72-366-0221, Fax: +81-72-367-2880, E-mail: mamoxyo45@gmail.com ORCID: https://orcid.org/0000-0001-7308-4311

(c) This is an Open Access article distributed under the terms of the Creative Commons Attribution Non-Commercial License (http://creativecommons.org/ licenses/by-nc/3.0) which permits unrestricted non-commercial use, distribution, and reproduction in any medium, provided the original work is properly cited. or abnormal MPD-related findings due to definite chronic pancreatitis, intraductal papillary mucinous neoplasms, and autoimmune pancreatitis were excluded. Patients with multiple stenoses were also excluded.

In this study, approximately $50 \%$ cases with FiCE were malignant. Moreover, the risk of pancreatic cancer was high in patients with FiCE when the pancreatic juice cytology result was Class 3 or higher. Therefore, the authors concluded that histological confirmation with pancreatic juice cytology is necessary before surgery to avoid unnecessary pancreatic resection. In our opinion, performing pancreatic juice cytology in patients with FiCE would aid in the early diagnosis of pancreatic cancer.

Studies have reported the diagnostic significance of serial pancreatic-juice aspiration cytologic examination (SPACE) using a nasopancreatic catheter that is endoscopically inserted via the papilla. ${ }^{3,4}$ In these reports, cytodiagnosis of the pancreatic juice conducted 5.3 times (range, 2-11 times) showed that the sensitivity, specificity, and accuracy for detecting pancreatic cancer were $100 \%, 83 \%$, and $95 \%$, respectively. However, similar studies have shown conflicting results. A reason for this discrepancy is a shortage of cytopathologists who are skilled and experienced in SPACE. Undoubtedly, it is essential to improve the diagnostic abilities of cytopathologists for early detection of pancreatic cancer.

As the authors noted, the study has a few limitations. It was a single-center analysis and patients with a benign diagnosis who did not undergo surgery were included. Moreover, patients with a definitive diagnosis of chronic pancreatitis were excluded. However, a definite diagnosis of chronic pancreatitis 
is often challenging. In general, mild chronic pancreatitis with pancreatic stenosis is not definitively diagnosed. Furthermore, chronic pancreatitis is a high-risk factor for pancreatic cancer and pancreatic stenosis of mild chronic pancreatitis may be malignant. In addition, there are early pancreatic cancers with pancreatic stenosis due to mass-like lesions. However, histopathologic findings may be masked by inflammation or fibrosis if the malignant cells are intraductal. In such cases, endoscopic ultrasound-guided fine-needle aspiration is not useful, and the lesion is diagnosed solely by pancreatic juice cytology. In this study, cases with mass-like lesions were excluded.

In the recent years, pancreatic duct changes and parenchymal atrophy have been identified as important initial findings of pancreatic cancer, highlighting the significance of FiCE in the early detection of suspected pancreatic cancer. ${ }^{5}$

In summary, there is no doubt that $\mathrm{FiCE}$ is an important determinant of early pancreatic cancer. However, FiCE requires SPACE, and SPACE needs experienced cytopathologists. Furthermore, findings other than FiCE, such as parenchymal atrophy, may also be predictive of early pancreatic cancer. Moreover, it should be noted that a single, localized stenosis of the MPD with a mass-like lesion may be indicative of early pancreatic cancer. Imaging techniques such as dynamic computed tomography, magnetic resonance imaging, and/or endoscopic ultrasound should be used for a comprehensive analysis of the morphological changes in the pancreas, the pancreatic duct, and the parenchyma to facilitate the early detection of tumors.
Lastly, further prospective studies with a larger sample size are recommended to confirm the predictive value of FiCE for detecting early pancreatic cancer. Multicenter prospective studies are warranted to analyze the outcomes in patients who have undergone surgery.

Conflicts of Interest

The authors have no financial conflicts of interest.

ORCID

Kentaro Yamao: https://orcid.org/0000-0003-2497-7740

Masatoshi Kudo: https://orcid.org/0000-0002-4102-3474

\section{REFERENCES}

1. Kanno Y, Koshita S, Ogawa T, et al. Predictive value of localized stenosis of the main pancreatic duct for early detection of pancreatic cancer. Clin Endosc 2019;52:588-597.

2. Egawa $\mathrm{S}$, Toma $\mathrm{H}$, Ohigashi $\mathrm{H}$, et al. Japan pancreatic cancer registry; 30th year anniversary: Japan Pancreas Society. Pancreas 2012;41:985992.

3. Iiboshi T, Hanada K, Fukuda T, Yonehara S, Sasaki T, Chayama K. Value of cytodiagnosis using endoscopic nasopancreatic drainage for early diagnosis of pancreatic cancer: establishing a new method for the early detection of pancreatic carcinoma in situ. Pancreas 2012;41:523-529.

4. Miyata T, Takenaka M, Omoto S, et al. A case of pancreatic carcinoma in situ diagnosed by repeated pancreatic juice cytology. Oncology 2017;93 Suppl 1:98-101.

5. Chu LC, Goggins MG, Fishman EK. Diagnosis and detection of pancreatic cancer. Cancer J 2017;23:333-342. 